\title{
Extensions of Effect Algebra Operations
}

\author{
Z. Riečanová, M. Zajac
}

\begin{abstract}
We study the set of all positive linear operators densely defined in an infinite-dimensional complex Hilbert space. We equip this set with various effect algebraic operations making it a generalized effect algebra. Further, sub-generalized effect algebras and interval effect algebras with respect of these operations are investigated.
\end{abstract}

Keywords: generalized effect algebra, effect algebra, Hilbert space, densely defined linear operators, extension of operations.

\section{Introduction and some basic definitions and facts}

The aim of this paper is to show that (generalized) effect algebras may be suitable and natural algebraic structures for sets of linear operators (including unbounded ones) densely defined on an infinitedimensional complex Hilbert space $\mathcal{H}$. In all cases, if the effect algebraic sum of operators $A, B$ is defined then it coincides with the usual sum of operators in $\mathcal{H}$.

Effect algebras were introduced by D. Foulis and M. K. Bennet in 1994 [2]. The prototype for the abstract definition of an effect algebra was the set $\mathcal{E}(\mathcal{H})$ (Hilbert space effects) of all selfadjoint operators between null and identity operators in a complex Hilbert space $\mathcal{H}$. If a quantum mechanical system is represented in the usual way by a complex Hilbert space $\mathcal{H}$, then self-adjoint operators from $\mathcal{E}(\mathcal{H})$ represent yes-no measurements that may be unsharp. The subset $\mathcal{P}(\mathcal{H})$ of $\mathcal{E}(\mathcal{H})$ consisting of orthogonal projections represents yes-no measurements that are sharp.

The abstract definition of an effect algebra follows the properties of the usual sum of operators in the interval $[0, I]$ (i.e. between null and identity operators in $\mathcal{H})$ and it is the following.

Definition 1 (Foulis, Bennet [2]) A partial algebra $(E ; \oplus, 0,1)$ is called an effect algebra if 0,1 are two distinguished elements and $\oplus$ is a partially defined binary operation on $E$ which satisfy the following conditions for any $x, y, z \in E$ :

(E1) $x \oplus y=y \oplus x$ if $x \oplus y$ is defined,

(E2) $(x \oplus y) \oplus z=x \oplus(y \oplus z)$ if one side is defined,

(E3) for every $x \in E$ there exists a unique $y \in E$ such that $x \oplus y=1$ (we put $x^{\prime}=y$ ),

(E4) If $1 \oplus x$ is defined then $x=0$.

Immediately in 1994 the study of generalizations of effect algebras (without the top element 1) was started by several authors (Foulis and Bennet [2], Kalmbach and Riečanová [4], Hedlíková and Pulmannová [3], Kôpka and Chovanec [5]). It was found that all these generalizations coincide, and their common definition is the following:

Definition 2 A generalized effect algebra $(E ; \oplus, 0)$ is a set $E$ with element $0 \in E$ and partial binary operation $\oplus$ satisfying for any $x, y, z \in E$ the conditions (GE1) $x \oplus y=y \oplus x$ if one side is defined,

(GE2) $(x \oplus y) \oplus z=x \oplus(y \oplus z)$ if one side is defined,

(GE3) If $x \oplus y=x \oplus z$ then $y=z$,

(GE4) If $x \oplus y=0$ then $x=y=0$,

(GE5) $x \oplus 0=x$ for all $x \in E$.

In every (generalized) effect algebra $E$ a partial order $\leq$ and a binary operation $\ominus$ can be introduced as follows: for any $a, b \in E, a \leq b$ and $b \ominus a=c$ iff $a \oplus c$ is defined and $a \oplus c=b$.

If the elements of a (generalized) effect algebra $E$ are positive linear operators in a given infinitedimensional complex Hilbert space, then $E$ is called an operator (generalized) effect algebra.

Throughout the paper we assume that $\mathcal{H}$ is an infinite-dimensional complex Hilbert space, i.e., a linear space with inner product $(\cdot, \cdot)$ which is complete in the induced metric. Recall that here for any $x, y \in \mathcal{H}$ we have $(x, y) \in \mathbb{C}$ (the set of all complex numbers) such that $(x, \alpha y+\beta z)=\alpha(x, y)+\beta(x, z)$ for all $\alpha, \beta \in \mathbb{C}$ and $x, y, z \in \mathcal{H}$. Moreover $(x, y)=\overline{(y, x)}$ and $(x, x) \geq 0$ with $(x, x)=0$ iff $x=0$. The term dimension of $\mathcal{H}$ in the following always means the Hilbertian dimension, i.e. the cardinality of any orthonormal basis of $\mathcal{H}$ (see [1, p. 44]).

For notions and results on Hilbert space operators we refer the reader to [1]. We will assume that the domains $D(A)$ of all considered linear operators $A$ are dense linear subspaces of $\mathcal{H}$ (in the metric topology induced by inner product). We say that operators $A$ are densely defined on $\mathcal{H}$. The set of all densely defined linear operators on $\mathcal{H}$ will be denoted by $\mathcal{L}(\mathcal{H})$. 
Recall that $A: D(A) \rightarrow \mathcal{H}$ is a bounded operator if there exists a real constant $C>0$ such that $\|A x\| \leq C\|x\|$ for all $x \in D(A)$. If $A$ is not bounded then it is called unbounded.

Let $T \in \mathcal{L}(\mathcal{H})$. Since $D(T)$ is dense in $\mathcal{H}$, for any $y \in \mathcal{H}$ there is at most one $y^{*} \in \mathcal{H}$ satisfying $(y, T x)=\left(y^{*}, x\right)$ for all $x \in D(T)$. This allows us to define the adjoint $T^{*}$ of $T$ by putting $D\left(T^{*}\right)=\left\{y \in \mathcal{H} \mid\right.$ there exists $y^{*} \in \mathcal{H}$ such that $(y, T x)=\left(y^{*}, x\right)$ for all $\left.x \in D(T)\right\}$ and $T^{*} y=y^{*}$. Operator $T$ is said to be self-adjoint if $T=T^{*}$. An operator $T \in \mathcal{L}(\mathcal{H})$ is called symmetric, if $T(x, y)=$ $(x, T y)$ for all $x, y \in D(A)$. It is well-known that this is equivalent with $(T x, x) \in \mathbb{R}$ for all $x \in D(T)$. Clearly every self-adjoint operator is symmetric but the converse need not hold for unbounded operators (see [1], p. 98).

Since every (generalized) effect algebra includes the zero element 0 as the least element of $E$, we will assume that all considered operators are positive (written $A \geq 0$ ). This means that $(A x, x) \geq 0$ for all $x \in D(A)$ and hence $A$ is also symmetric, i.e. $(A x, y)=(x, A y)$ for all $x, y \in D(A)$ (see [1, pp. 68 and 94]). For two operators $A: D(A) \rightarrow \mathcal{H}$ and $B: D(B) \rightarrow \mathcal{H}$ we write $A \subset B$ iff $D(A) \subset D(B)$ and $A x=B x$ for all $x \in D(A)$. Then $B$ is called an extension of $A$.

We show some examples of partial binary operations (sums) on the set $\mathcal{V}(\mathcal{H})$ of all positive linear operators densely defined on infinite-dimensional Hilbert space $\mathcal{H}$. Our main goal is to study the properties of sub-generalized effect algebras and effect algebras being intervals in $\mathcal{V}(\mathcal{H})$ if $\mathcal{V}(\mathcal{H})$ is equipped with two different partial sums such that one of them is an extension of the other.

\section{Some properties of unbounded operators in complex Hilbert spaces}

Before defining (generalized) effect algebras consisting of operators $A \in \mathcal{L}(\mathcal{H})$, we review some necessary results on Hilbert space operators [1, Chapter 4].

Theorem 3 Let $D_{1} \subset D_{2} \subset \mathcal{H}$ be linear subspaces, $\overline{D_{1}}=\overline{D_{2}}=\mathcal{H}$. Let $A \in \mathcal{L}(\mathcal{H}), D(A)=D_{2}$ and its restriction $A_{1}=A \mid D_{1}=0$ and $(A x, x) \in \mathbb{R}$ for all $x \in D_{2}$. Then $A=0$.

Proof. If $A \neq 0$, then $\exists e \in D_{2} \backslash D_{1}$ for which $A e \neq 0$. For all $d \in D_{1}$ and all $\lambda \in \mathbb{C}$

$$
\begin{aligned}
& A d=0, d+\lambda e \in D_{2}, \\
& (A(d+\lambda e), d+\lambda e)=\bar{\lambda}(A e, d)+|\lambda|^{2}(A e, e) \in \mathbb{R} .
\end{aligned}
$$

Since $D_{1}^{\perp}=\{0\}$ and $A e \neq 0$, we can choose $d_{1} \in D_{1}$ for which $\left(A e, d_{1}\right) \neq 0$. Then, by $(1)$,

$$
\forall \lambda \in \mathbb{C}, \bar{\lambda}\left(A e, d_{1}\right)+|\lambda|^{2}(A e, e) \in \mathbb{R} .
$$

Since the second summand is real, the first one must be real for all $\lambda \in \mathbb{C}$. However, this is possible only if $\left(A e, d_{1}\right)=0$, a contradiction.

Corollary 4 If $A \in \mathcal{L}(\mathcal{H}), D=D(A) \neq \mathcal{H}$. is a symmetric bounded operator and $B \in \mathcal{L}(\mathcal{H})$ is its symmetric extension, then $B$ is also bounded.

Proof. Let $B$ be a proper symmetric extension of $A$ and let $\widetilde{A}$ be the unique bounded extension of $A$. Then $(B-\widetilde{A}) \mid D=0$ and, by Theorem 3, $(B-\widetilde{A}) \mid D(B)=0$. It follows that $B=B-\widetilde{A} \mid D(B)+$ $\widetilde{A}|D(B)=\widetilde{A}| D(B)$ is a bounded linear operator.

Theorem 5 Let $A \in \mathcal{L}(\mathcal{H})$ and there exists

$$
k>0 \text { such that } \forall x \in D(A) \quad|(A x, x)| \leq k\|x\|^{2}
$$

Then $A$ is bounded.

Proof. Let there exist $k>0$ such that

$$
\forall x \in D(A) \quad|(A x, x)| \leq k\|x\|^{2}
$$

Using the polarization identity:

$$
\begin{aligned}
& (A x, y)= \\
& \frac{1}{4}\{(A(x+y), x+y)-(A(x-y), x-y)+ \\
& i[(A(i x+y), i x+y)-(A(i x-y), i x-y)]\},
\end{aligned}
$$

we obtain for any $x, y \in D(A),\|x\|=\|y\|=1$, that

$|(A x, y)| \leq$

$\frac{1}{4}\{|(A(x+y), x+y)|+|(A(x-y), x-y)|+$

$|(A(i x+y), i x+y)|+|(A(i x-y), i x-y)|\} \leq$

$\frac{k}{4}\left\{\|x+y\|^{2}+\|x-y\|^{2}+\|i x+y\|^{2}+\|i x-y\|^{2}\right\}=$

$\frac{k}{4}\{(x+y, x+y)+(x-y, x-y)+$

$(i x+y, i x+y)+(i x-y, i x-y)\}=$

$\frac{k}{4}\left\{2\|x\|^{2}+2\|y\|^{2}+2\|i x\|^{2}+2\|y\|^{2}\right\}=$

$k\left(\|x\|^{2}+\|y\|^{2}\right)=2 k$.

This means that the quadratic form $(A x, y)$ is bounded, i.e.

$$
\forall x, y \in D(A) \quad|(A x, y)| \leq 2 k\|x\|\|y\| .
$$

It follows that for any fixed $x \in D(A)$ the linear functional $\varphi(y)=(A x, y)$ is bounded and defined on $D(A)$ therefore $\tilde{\varphi}: \overline{D(A)}=\mathcal{H} \rightarrow \mathbb{C}, \widetilde{\varphi}(y)=(A x, y)$ for all $y \in \mathcal{H}$ is its unique bounded linear extension and $\|\widetilde{\varphi}\|=\|\varphi\| \leq 2 k\|x\|$. Now putting $y=A x$ we obtain, by (3),

$$
\begin{aligned}
\|A x\|^{2} & =(A x, A x)=\widetilde{\varphi}(A x) \leq 2 k\|x\|\|A x\| \Longrightarrow \\
\|A x\| & \leq 2 k\|x\| .
\end{aligned}
$$


Corollary 6 Let $A, B$ be nonnegative densely defined linear operators having the same domain $D$. If $A+B$ is bounded then both $A, B$ are bounded.

Proof. It suffices to observe that

$$
\begin{aligned}
0 \leq & (A x, x) \leq(A x, x)+(B x, x)= \\
& ((A+B) x, x) \leq\|A+B\|\|x\|^{2}
\end{aligned}
$$

and then, by Theorem $5, A$ is bounded. By the same reasoning we obtain that $B$ is bounded.

\section{Extensions of effect algebra operations}

It is well known that if a set $E$ includes two distinguished elements 0,1 then there may exist more than one partial binary operations $\oplus$ on $E$ making $E$ an effect algebra.

Example 7 Let $E=\{0, a, b, 1\}$ and let us define operations $\oplus_{1}, \oplus_{2}$ on $E$ as follows:

$\oplus_{1}: a \oplus_{1} b=b \oplus_{1} a=1$ and $0 \oplus_{1} x=x \oplus_{1} 0=x$ for all $x \in E$,

$\oplus_{2}: a \oplus_{2} a=b \oplus_{2} b=1$ and $0 \oplus_{2} x=x \oplus_{2} 0=x$ for all $x \in E$.

Then $\left(E ; \oplus_{1}, 0,1\right),\left(E ; \oplus_{2}, 0,1\right)$ are effect algebras with the same set of elements. On the other hand $\left(E ; \oplus_{1}, 0,1\right)$ is a Boolean algebra, while $\left(E ; \oplus_{2}, 0,1\right)$ is a horizontal sum of two chains $\left\{0, a, a \oplus_{2} a=1\right\}$ and $\left\{0, b, b \oplus_{2} b=1\right\}$, hence in this case elements $a, b$ are noncompatible.

We obtain special cases of two operations $\oplus_{1} \neq \oplus_{2}$ on the same underlying set $E$, if $\oplus_{2}$ extends $\oplus_{1}$ :

Definition 8 Let $\left(E ; \oplus_{1}, 0\right)$ and $\left(E ; \oplus_{2}, 0\right)$ be generalized effect algebras. We say that the operation $\oplus_{2}$ extends $\oplus_{1}$ (written $\oplus_{1} \subset \oplus_{2}$ ) if for any $a, b \in E$ the existence of $a \oplus_{1} b$ implies that $a \oplus_{2} b$ exists and $a \oplus_{2} b=a \oplus_{1} b$.

Lemma 9 Let $E_{1}=\left(E ; \oplus_{1}, 0\right), E_{2}=\left(E ; \oplus_{2}, 0\right)$ be generalized effect algebras and $\oplus_{1} \subset \oplus_{2}$. Then

(i) If $G \subseteq E$ is a sub-generalized effect algebra of $E_{2}$, then $G$ is also a sub-generalized effect algebra of $E_{1}$.

(ii) If $\leq_{1}$ and $\leq_{2}$ are the partial orders on $E$ derived from $\oplus_{1}$ and $\oplus_{2}$, respectively, then, for $a, b \in E$, if $a \leq_{1} b$ then $a \leq_{2} b$.

(iii) For intervals in $E_{1}, E_{2}$ the following inclusion holds:

$[0, q]_{E_{1}} \subseteq[0, q]_{E_{2}}$ for any nonzero $q \in E$.

Proof. The proof obviously follows from the fact that, for any $a, b \in E$, the existence of $a \oplus_{1} b$ implies $a \oplus_{2} b=a \oplus_{1} b$. Let us prove, e.g., (i): Let $a, b, c \in E$ with $a \oplus_{1} b=c$ and assume that at least two out of elements $a, b, c$ are in $G$. Since $\oplus_{1} \subset \oplus_{2}$ implies $a \oplus_{1} b=c=a \oplus_{2} b=c$ and $G$ is a sub-effect algebra of $E_{2}$ we obtain $a, b, c \in G$. Hence $G$ is a sub-generalized effect algebra of $E_{1}$.

The following example shows that the converses of assertions (i)-(iii) do not hold.

Example 10 Let $E=\{0,1,2, \ldots\}$ and $G=$ $\{0,1,2,4,6, \ldots\}$. Define the partial binary operations $\oplus_{1}$ and $\oplus_{2}$ for $a, b \in E$

$$
\begin{aligned}
& a \oplus_{1} b=\left\{\begin{array}{l}
a+b, \text { if } a=0 \text { or both } a, b \text { are even, } \\
\text { not defined, otherwise, }
\end{array}\right. \\
& a \oplus_{2} b=a+b \text { for all } a, b \in E
\end{aligned}
$$

and let $\leq_{1}$ and $\leq_{2}$ be the corresponding derived partial orders. Then obviously $\oplus_{1} \subset \oplus_{2}$ and

(i) $E_{1}=\left(E, \oplus_{1}, 0\right)$ and $E_{2}=\left(E, \oplus_{2}, 0\right)$ are generalized effect algebras.

(ii) $G$ is a sub-generalized effect algebra of $E_{1}$.

(iii) $G$ is not a sub-generalized effect algebra of $E_{2}$.

(iv) There exist $a, b \in E$ for which $a \leq_{2} b$ but $a \leq_{1} b$.

(v) There exist $q \in E$ for which $[0, q]_{E_{2}} \nsubseteq[0, q]_{E_{1}}$. Let us prove conditions (i) $-(\mathrm{v})$.

(i) Let us show that $\oplus_{1}$ is associative. To show this, suppose that for $a, b, c \in E$ the sum $\left(a \oplus_{1} b\right) \oplus_{1} c$ exists. First, if $c=0$ then $\left(a \oplus_{1} b\right) \oplus_{1} c=a \oplus_{1} b=$ $a \oplus_{1}\left(b \oplus_{1} c\right)$. Next, if $c \neq 0$ then either $a=b=0$ or both $c$ and $a+b$ are even. If $a=b=0$ then $\left(a \oplus_{1} b\right) \oplus_{1} c=a \oplus_{1}\left(b \oplus_{1} c\right)$ is obvious. $a+b$ is even if both $a, b$ are odd, but this is impossible because $a \oplus_{1} b$ exists. So, both $a, b$ are even and, then, again, $\left(a \oplus_{1} b\right) \oplus_{1} c=a \oplus_{1}\left(b \oplus_{1} c\right)$ is obvious. The rest of the proof of (i) is obvious.

(ii) Suppose that $a, b, c \in E$ satisfy $a \oplus_{1} b=c$. If $a, b \in G$ then clearly $c \in G$ as well. If $a, c \in G$ and $a=0$, then $b=c$, hence $b \in G$. $a, c \in G$ are nonzero then both are even and then $b$ is even, as well. So, if two elements out of $a, b, c$ are in $G$, then all three are in $G$. This proves (ii).

(iii) Put $a=1, b=2$. Now $a, b \in G$ and $a \oplus_{2} b=3 \notin G$ shows that $G$ is not a sub-generalized effect algebra of $E_{2}$.

(v) Clearly, $[0, q]_{E_{1}}=\{0, q\}$ for any odd $q$. So, e.g. $[0,3]_{E_{2}}=\{0,1,2,3\} \nsubseteq[0,3]_{E_{1}}$.

(iv) obviously (v) implies (iv).

\section{Extensions of operator effect algebra operations}

We introduce examples of operator generalized effect algebra with the same set of elements and different operations. Moreover, we consider intervals in these algebras. 
A generalized effect algebra whose elements are positive linear operators on a complex Hilbert space $\mathcal{H}$ is called an operator generalized effect algebra.

Definition 11 Assume that $\mathcal{H}$ is an infinitedimensional Hilbert space and that $A \in \mathcal{L}(\mathcal{H})$ is positive. Let $\mathcal{D}$ denote the set of all dense linear subspaces of $\mathcal{H}$ and

(i) $\mathcal{V}(\mathcal{H})=\{A \in \mathcal{L}(\mathcal{H}) \mid A \geq 0, D(A)=\mathcal{H}$ if $A$ is bounded and $D(A) \in \mathcal{D}$ if $A$ is unbounded .

(ii) $\mathcal{G}_{D}(\mathcal{H})=\{A \in \mathcal{V}(\mathcal{H}) \mid A$ is bounded or $D(A)=$ $D$ if $A$ is unbounded $\}, D \in \mathcal{D}$.

(iii) Let $\oplus$ be a partial binary operation on $\mathcal{V}(\mathcal{H})$ defined by:

For $A, B \in \mathcal{V}(\mathcal{H}), A \oplus B$ is defined and $A \oplus B=$ $A+B$ (the usual sum) iff at least one out of operators $A, B$ is bounded. The triple $(\mathcal{V}(\mathcal{H}) ; \oplus, 0)$ will be denoted by $\mathcal{V}(\mathcal{H})$ for short.

(iv) Let $\oplus_{\mathcal{D}}$ be a partial binary operation on $\mathcal{V}(\mathcal{H})$ defined by:

For $A, B \in \mathcal{V}(\mathcal{H}), A \oplus_{\mathcal{D}} B$ is defined and $A \oplus_{\mathcal{D}}$ $B=A+B$ (the usual sum) iff either at least one out of $A, B$ is bounded, or $D(A)=D(B) \in \mathcal{D}$ if both are unbounded. The triple $\left(\mathcal{V}(\mathcal{H}) ; \oplus_{\mathcal{D}}, 0\right)$ will be denoted by $\mathcal{V}_{\mathcal{D}}(\mathcal{H})$ for short.

From the above definition it is clear that $\mathcal{V}(\mathcal{H})=$ $\bigcup\left\{\mathcal{G}_{D}(\mathcal{H}) \mid D \in \mathcal{D}\right\}$ and for $D_{1} \neq D_{2}$ it holds $\mathcal{G}_{D_{1}}(\mathcal{H}) \cap \mathcal{G}_{D_{1}}(\mathcal{H})=\mathcal{B}^{+}(\mathcal{H})=\{A \in \mathcal{V}(\mathcal{H}) \mid A$ is bounded with $D(A)=\mathcal{H}$ \}.

Moreover, for any $A, B \in \mathcal{V}(\mathcal{H})$ if $A \oplus B$ is defined then $A \oplus_{\mathcal{D}} B$ is defined and $A \oplus_{\mathcal{D}} B=A \oplus B=A+B$. Hence $\oplus \subset \oplus_{\mathcal{D}}$.

Recently in $[6,8]$ the following theorems were proved.

Theorem 12 [6] For $\mathcal{V}(\mathcal{H})$ from Definition 11 it holds:

(i) $(\mathcal{V}(\mathcal{H}) ; \oplus, 0)$ is a generalized effect algebra.

(ii) If $\mathcal{S}_{p}(\mathcal{H})=\left\{A \in \mathcal{V}(\mathcal{H}) \mid A=A^{*}\right\}$ is equipped with $\left.\oplus\right|_{\mathcal{S}_{p}(\mathcal{H})}$, i.e., for $A, B \in \mathcal{S}_{p}(\mathcal{H})$ there exists $\left.A \oplus\right|_{\mathcal{S}_{p}(\mathcal{H})} B=A \oplus B$ iff there exists $A \oplus$ $B$ in $\mathcal{V}(\mathcal{H})$, then $\left(\mathcal{S}_{p}(\mathcal{H}) ;\left.\oplus\right|_{\mathcal{S}_{p}(\mathcal{H})}, 0\right)$ is a subgeneralized effect algebra of $(\mathcal{V}(\mathcal{H}) ; \oplus, 0)$.

Theorem 13 [8] Using the notation from Definition 11 we obtain:

(i) $\left(\mathcal{V}(\mathcal{H}) ; \oplus_{\mathcal{D}}, 0\right)$ is a generalized effect algebra.

(ii) Let $D \in \mathcal{D}$ be a fixed dense subspace of $\mathcal{H}$. Let $\mathcal{G}_{\mathcal{D}, D}(\mathcal{H})=\{A \in \mathcal{V}(\mathcal{H}) \mid$ either $A$ is bounded with $D(A)=\mathcal{H}$ or $A$ is unbounded with $D(A)=D\}$. Let for $A, B \in \mathcal{G}_{\mathcal{D}, D}(\mathcal{H})$ the sum $\left.A \oplus_{\mathcal{D}}\right|_{\mathcal{G}_{\mathcal{D}, D}(\mathcal{H})} B=A \oplus_{\mathcal{D}} B$ if there exists $A \oplus_{\mathcal{D}}$ $B \in \mathcal{G}_{\mathcal{D}, D}(\mathcal{H})$, otherwise $\left.A \oplus_{\mathcal{D}}\right|_{\mathcal{G}_{\mathcal{D}, D}(\mathcal{H})} B$ is not defined. Then $\left(\mathcal{G}_{\mathcal{D}, D}(\mathcal{H}) ;\left.A \oplus_{\mathcal{D}}\right|_{\mathcal{G}_{\mathcal{D}, D}(\mathcal{H})}, 0\right)$ is a sub-generalized effect algebra of $\left(\mathcal{V}(\mathcal{H}), \oplus_{\mathcal{D}}, 0\right)$.

Now, because $\oplus \subset \oplus_{\mathcal{D}}$ on $\mathcal{V}(\mathcal{H})$ and, for every fixed $D \in \mathcal{D}, \mathcal{G}_{\mathcal{D}, D}(\mathcal{H})$ is a sub-generalized effect algebra of
$\left(\mathcal{V}(\mathcal{H}) ; \oplus_{\mathcal{D}}, 0\right)$ we obtain that $\mathcal{G}_{\mathcal{D}, D}(\mathcal{H})$ is also a subgeneralized effect algebra of $(\mathcal{V}(\mathcal{H}) ; \oplus, 0)$. Clearly, the intersection of two sub-generalized effect algebras of $(\mathcal{V}(\mathcal{H}) ; \oplus, 0)$ is again its sub-generalized effect algebra. Thus we obtain the following corollary of Theorems 12 and 13 .

Theorem 14 Let for every fixed $D \in \mathcal{D}, \mathcal{S}_{p, D}(\mathcal{H})=$ $\left\{\mathcal{S}_{p}(\mathcal{H}) \cap \mathcal{G}_{\mathcal{D}, D}(\mathcal{H})=\left\{A \in \mathcal{V}(\mathcal{H}) \mid A=A^{*}\right.\right.$ and either $D(A)=\mathcal{H}$, if $A$ is bounded, or $D(A)=D$ if $A$ is unbounded $\}$. Let $\left.\oplus\right|_{\mathcal{S}_{p, D}(\mathcal{H})}$ on $\mathcal{S}_{p, D}(\mathcal{H})$ be defined as follows: For $A, B \in \mathcal{S}_{p, D}(\mathcal{H}),\left.A \oplus\right|_{\mathcal{S}_{p, D}(\mathcal{H})} B=$ $A \oplus B$ iff $A \oplus B$ is defined in $\mathcal{V}(\mathcal{H})$. Then $\left(\mathcal{S}_{p, D}(\mathcal{H}) ;\left.\oplus\right|_{\mathcal{S}_{p, D}(\mathcal{H})}, 0\right)$ is a sub-generalized effect algebra of $\left(\mathcal{S}_{p}(\mathcal{H}) ; \oplus, 0\right)$.

The above observations show that generalized effect algebra $\mathcal{V}_{\mathcal{D}}(\mathcal{H})$ is a pasting of its sub-generalized effect algebras $\mathcal{G}_{\mathcal{D}, D}(\mathcal{H})$ through the sub-generalized effect algebra $\mathcal{B}^{+}(\mathcal{H})=\{A \in \mathcal{V}(\mathcal{H}) \mid A$ is bounded $\}$. Consequently, the generalized effect algebra $\mathcal{S}_{p}(\mathcal{H})$ is a pasting of its sub-generalized effect algebras $\mathcal{S}_{p, D}(\mathcal{H})$ through the sub-generalized effect algebra $\mathcal{B}^{+}(\mathcal{H})$. More precisely:

\section{Theorem 15 (Pasting Theorem)}

(i) For generalized effect algebra $\mathcal{V}_{\mathcal{D}}(\mathcal{H})=\left\{\mathcal{V}(\mathcal{H}) ; \oplus_{\mathcal{D}}, 0\right)$ and its sub-generalized effect algebras $\mathcal{G}_{\mathcal{D}, D}(\mathcal{H}), D \in \mathcal{D}$, and $\mathcal{B}^{+}(\mathcal{H})$

(1) $\mathcal{G}_{\mathcal{D}, D_{1}}(\mathcal{H}) \cap \mathcal{G}_{\mathcal{D}, D_{2}}(\mathcal{H})=\mathcal{B}^{+}(\mathcal{H})$ for every $D_{1}, D_{2} \in \mathcal{D}, D_{1} \neq D_{2}$.

(2) $\mathcal{V}_{\mathcal{D}}(\mathcal{H})=\bigcup\left\{\mathcal{G}_{\mathcal{D}, D}(\mathcal{H}) \mid D \in \mathcal{D}\right\}$.

(ii) For generalized effect algebra $\mathcal{S}_{p}(\mathcal{H})$ and its subgeneralized effect algebras $\mathcal{S}_{p, D}(\mathcal{H}), D \in \mathcal{D}$

(1) $\mathcal{S}_{p D_{1}}(\mathcal{H}) \cap \mathcal{S}_{p D_{2}}(\mathcal{H})=\mathcal{B}^{+}(\mathcal{H})$ for every $D_{1}, D_{2} \in \mathcal{D}, D_{1} \neq D_{2}$.

(2) $\mathcal{S}_{p}(\mathcal{H})=\bigcup\left\{\mathcal{S}_{p, D}(\mathcal{H}) \mid D \in \mathcal{D}\right\}$.

Remark 16 It is worth noting that the operations $\oplus, \oplus_{\mathcal{D}}$ are the usual sum of operators in $\mathcal{H}$, but only partially applied on pairs $A, B \in \mathcal{V}(\mathcal{H})$. Therefore all effect algebra operations $\oplus, \oplus_{\mathcal{D}},\left.\oplus\right|_{\mathcal{S}_{p}(\mathcal{H})}$, $\left.\oplus_{\mathcal{D}}\right|_{\mathcal{G}_{\mathcal{D}, D}(\mathcal{H})},\left.\oplus\right|_{\mathcal{S}_{p, D}(\mathcal{H})}$ coincide with the usual sum of operators $A+B$ if the corresponding effect algebra sum of $A$ and $B$ exists.

\section{Intervals in generalized effect algebras of self-adjoint operators}

Assume that $(E ; \oplus, 0)$ is a generalized effect algebra. For any $q \in E, q \neq 0$, let $[0, q]_{E}=\{a \in E \mid$ there exists $b \in E$ with $a \oplus b=q\}$ be an interval in $(E ; \oplus, 0]$. We will denote by $\left.\oplus\right|_{[0, q]_{E}}$ the partial binary operation on $[0, q]_{E}$ defined as follows: 
For $a, b \in[0, q]_{E}$ the sum $\left.a \oplus\right|_{[0, q]_{E}} b=a \oplus b$ iff $a \oplus b$ is defined in $E$ and $a \oplus b \in[0, q]_{E}$. It is known that $[0, q]_{E}$ equipped with $\left.\oplus\right|_{[0, q]_{E}}$ is an effect algebra (see, e.g. [7]).

In this way, for all nonzero $Q \in \mathcal{V}(\mathcal{H})$, we obtain the operator effect algebras

$$
\left([0, Q]_{\mathcal{V}(\mathcal{H})} ;\left.\oplus\right|_{[0, Q]_{\mathcal{V}(\mathcal{H})}}, 0, Q\right)
$$

and

$$
\left([0, Q]_{\mathcal{V}_{\mathcal{D}}(\mathcal{H})} ;\left.\oplus_{\mathcal{D}}\right|_{[0, Q]]_{\mathcal{D}}(\mathcal{H})}, 0, Q\right)
$$

(see [8]). By definition of $\oplus, \oplus_{\mathcal{D}}$ and the results of Section 2, it is clear that for $Q \in \mathcal{V}(\mathcal{H})$ with $D(Q)=D \in \mathcal{D}$ we have $[0, Q]_{\mathcal{V}(\mathcal{H})} \subset \mathcal{G}_{D}(\mathcal{H})$ and

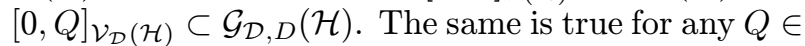
$\mathcal{S}_{p}(\mathcal{H}), Q \neq 0, D(Q)=D$. Namely, $[0, Q]_{\mathcal{S}_{p}(\mathcal{H})}=$ $[0, Q]_{\mathcal{S}_{p, D}(\mathcal{H})}$ and $\left([0, Q]_{\mathcal{S}_{p, D}(\mathcal{H})} ;\left.\oplus\right|_{[0, Q]_{\mathcal{S}_{p, D}(\mathcal{H})}}, 0, Q\right)$, $D \in \mathcal{D}$, are effect algebras of positive self-adjoint operators $A \leq_{D} Q$, where $\leq_{D}$ is the partial order on $\mathcal{S}_{p}(\mathcal{H})$ derived from operation $\left.\oplus\right|_{\mathcal{S}_{p, D}(\mathcal{H})}$. Since $D(Q)=D$ is dense in $\mathcal{H}$, the next Theorem 18 about states on intervals is $\mathcal{S}_{p}(\mathcal{H})$ (hence in $\mathcal{S}_{p, D}(\mathcal{H})$ ) can be proved by the same argument as Theorem 7 in [8] for states on intervals in $\mathcal{G}_{\mathcal{D}, D}(\mathcal{H})$.

Definition 17 Let $E$ be an effect algebra.

(i) A map $\omega: E \rightarrow[0,1]$ is called a state on $E$ if

1. $\omega(0)=0, \omega(1)=1$,

2. $\omega(a \oplus b)=\omega(a)+\omega(b)$ for all $a, b \in E$ with $a \oplus b$ defined in $E$.

3. A state $\omega$ is faithful if $\omega(a)=0$ implies $a=0$.

4. A set $\mathcal{M}$ of states is called an ordering set of states on $E$ if $a \leq b$ iff $\omega(a) \leq \omega(b)$ for all $\omega \in \mathcal{M}, a, b \in E$.

Theorem 18 Let $D \in \mathcal{D}$ and $Q \in \mathcal{S}_{p, D}(\mathcal{H}), Q \neq 0$. Then

(i) There exists $\tilde{x} \in D(Q)$ such that $c_{\tilde{x}}=(Q \tilde{x}, \tilde{x})>$ 0 .

(ii) The mapping $\omega_{\tilde{x}}:[0, Q]_{\mathcal{S}_{p, D}(\mathcal{H})} \rightarrow[0,1] \subset \mathbb{R}$ given by $\omega_{\tilde{x}}(A)=\frac{1}{c_{\tilde{x}}}(A \tilde{x}, \tilde{x})$ for every $A \in$ $[0, Q]_{\mathcal{S}_{p, D}(\mathcal{H})}$ is a state.

(iii) If $D_{0}=\left\{x \in D(Q) \mid c_{x}=(Q x, x)>0\right\}$ then $\mathcal{M}=\left\{\omega_{x} \mid x \in D_{0}\right\}$ is an ordering set of states on $[0, Q]_{\mathcal{S}_{p, D}(\mathcal{H})}$.

(iv) If $\mathcal{H}$ is separable, then there exists a faithful state $\omega:[0, Q]_{\mathcal{S}_{p, D}(\mathcal{H})} \rightarrow[0,1]$.

\section{Acknowledgement}

Supported by grants VEGA 1/0297/11 and VEGA $1 / 0021 / 10$ of the Ministry of Education of the Slovak Republic.

\section{References}

[1] Blank, J., Exner, P., Havlíček, M.: Hilbert Space Operators in Quantum Physics (second edition). Springer, 2008.

[2] Foulis, D. J., Bennet, M. K.: Effect algebras and unsharp quantum logics, Found. Phys. 24 (1994), 1331-1352.

[3] Hedlíková, J., Pulmannová, S.: Generalized difference posets and orthoalgebras, Acta Math. Univ. Comenianae 45 (1996), 247-279.

[4] Kalmbach, G., Riečanová, Z.: An axiomatization for abelian relative inverses, Demonstratio Math. 27 (1996), 769-780.

[5] Kôpka, F., Chovanec, F.: D-posets, Math. Slovaca 44 (1994), 21-34.

[6] Riečanová, Z.: Effect algebras of positive selfadjoint operators densely defined on Hilbert spaces, preprint.

[7] Riečanová, Z.: Subalgebras, intervals and central elements of generalized effect algebras. International Journal of Theoretic Physics 38 (1999), 3209-3220.

[8] Riečanová, Z., Zajac, M., Pulmannová, S.: Effect algebras of positive linear operators densely defined on Hilbert spaces, Reports of Mathematical Physics, to appear.

Zdenka Riečanová

E-mail: zdenka.riecanova@stuba.sk

M. Zajac

E-mail: zajacm@stuba.sk

Department of Mathematics

Faculty of Electrical Engineering

and Information Technology STU

Ilkovičova 3, SK-81219 Bratislava 